\title{
OSCILLATION OF UNBOUNDED SOLUTIONS OF FOURTH ORDER NEUTRAL DYNAMIC EQUATIONS VIA TAYLOR'S FORMULA
}

\section{A. TRIPATHY *}

Abstract. This paper deals with the unbounded oscillation properties of nonlinear fourth order neutral delay dynamic equations of the form

$$
\left(p(t)\left((u(t)+r(t) u(\alpha(t)))^{\Delta^{2}}\right)\right)^{\Delta^{2}}+g(t) G(u(\beta(t)))-h(t) H(u(\gamma(t)))=0
$$

on an arbitrary time scales $\mathbb{T}$, under the assumptions

$$
\int_{t_{0}}^{\infty} \frac{t}{p(t)} \Delta t=\infty \text { or } \int_{t_{0}}^{\infty} \frac{t}{p(t)} \Delta t<\infty, t_{0}>0
$$

for various ranges of $r(t)$ with $|r(t)|<\infty$.

Key Words. Oscillation; Nonlinear; Delay dynamic equations; Time scales.

AMS(MOS) subject classification. 34K11, 34A10, 39A99

1. Introduction. In [14] and [15], Panigrahi and Reddy have discussed the oscillation and asymptotic properties of solutions of functional dynamic equations of type

$$
\left(p(t)(u(t)+r(t) u(\alpha(t)))^{\Delta^{2}}\right)^{\Delta^{2}}+g(t) G(u(\beta(t)))=0,
$$

where $g, p \in C_{r d}\left(\mathbb{T}, \mathbb{R}_{+}\right), \alpha, \beta \in C_{r d}(\mathbb{T}, \mathbb{T})$ such that $\alpha(t) \leq t, \beta(t) \leq t$, and $\lim _{t \rightarrow \infty} \alpha(t)=\infty=\lim _{t \rightarrow \infty} \beta(t), G \in C(\mathbb{R}, \mathbb{R})$ is a continuous function with

* Depatment of Mathematics, Sambalpur University, Sambalpur-768019, INDIA 
the property $x G(x)>0$ for $x \neq 0$, and $r \in C_{r d}(\mathbb{T}, \mathbb{R})$ such that $|r(t)|<\infty$, under the assumptions

$\left(A_{0}\right) \quad \int_{t_{0}}^{\infty} \frac{t}{p(t)} \Delta t<\infty, \quad t_{0}>0$

and

$\left(A_{00}\right) \quad \int_{t_{0}}^{\infty} \frac{t}{p(t)} \Delta t=\infty, \quad t_{0}>0$.

In time scale calculus, we take the sign convention $\Delta$ as the differential operator for right-scattered points on $\mathbb{T}$ with the property $\Delta^{k}=\Delta \Delta^{k-1}$ and $C_{r d}$ as the continuous for right-scattered and right-dense points on $\mathbb{T}$.

When we are concerned with oscillation of all solutions of (1.1), immediately we go for the works [19] and [20] by Tripathy under two sorts of assumptions $\left(A_{00}\right)$ and $\left(A_{0}\right)$ respectively. Similarly, in an another work [8] of Grace and Zafer we find an all solution oscillatory problem for the dynamic equations

$$
\left(p(t)\left((u(t)+r(t) u(\alpha(t)))^{\Delta^{3}}\right)^{\mu_{1}}\right)^{\Delta}+g(t) u^{\mu_{2}}(\beta(t))=0
$$

under the following restrictions:

(i) $\mu_{1}$ and $\mu_{2}$ are the quotient of odd positive integers such that $\mu_{2} \leq \mu_{1}$,

(ii) $|r(t)| \leq 1, \quad$ (iii) $\int_{t_{0}}^{\infty} p^{-\frac{1}{\mu_{1}}}(t) \Delta t=\infty, \quad t_{0}>0$.

And they have established the results with the helping tool

$$
h_{n+1}(t, s)=\int_{s}^{t} h_{n}(\tau, s) \Delta \tau, n=1,2,3, \ldots ; h_{0}(t, s)=1,
$$

where $h_{n}: \mathbb{T} \times \mathbb{T} \rightarrow \mathbb{R}$ which is known as Taylor monomials, but the neutral coefficient is too restrictive. Upon the choice of same problem, it is not hard to review the work for other ranges of $r(t)$ with respect to the works [19] and [20] even if,

$$
\int_{t_{0}}^{\infty} p^{-\frac{1}{\mu_{1}}}(t) \Delta t<\infty, \quad t_{0}>0
$$

also. However, there are no results in case when we consider $g^{+}(t)=$ $\max \{0, g(t)\}$ and $g^{-}(t)=\max \{0,-g(t)\}$ for (1.1) which takes the form:

$$
\begin{gathered}
\left(p(t)(u(t)+r(t) u(\alpha(t)))^{\Delta^{2}}\right)^{\Delta^{2}}+ \\
+g^{+}(t) G(u(\beta(t)))-g^{-}(t) G(u(\beta(t)))=0 .
\end{gathered}
$$

The objective of this work is to go for an all solution oscillatory problem for the functional equation 


$$
\begin{gathered}
\left(p(t)(u(t)+r(t) u(\alpha(t)))^{\Delta^{2}}\right)^{\Delta^{2}}+ \\
+g(t) G(u(\beta(t)))-h(t) H(u(\beta(t)))=0
\end{gathered}
$$

for different ranges of $r(t)$. In (1.3), we impose conditions upon $H$ as follows:

$$
H \in C(\mathbb{R}, \mathbb{R}), x H(x)>0 \text { for } x \neq 0, H \text { is bounded. }
$$

Graef et al.[9] have established the sufficient conditions for oscillation and asymptotic behaviour of solutions of (1.3) by means of key assumption $\left(A_{00}\right)$ but, with a slight deviation (1.3) has been studied by Panigrahi et al.[16] under the assumption

$$
\int_{t_{0}}^{\infty} \frac{\sigma(t)}{p(t)} \Delta t<\infty, \quad t_{0}>0 .
$$

We notice that (1.3) can be studied by means of $\left(A_{0}\right)$ also. Unlike the methods of [9] and [16], the purpose of this work is to study (1.3) via Taylor's theorem in the sense of Time scales(see for e.g [4], [5]). For our work, we assume the following assumptions on $G, \alpha$ and $\beta$ :

$\left(A_{1}\right) G(x y) \geq G(x) G(y)$, for $x, y \in \mathbb{R}$ and $x, y>0$, $\left(A_{2}\right) G(-x)=-G(x)$, for $x \in \mathbb{R}$, $\left(A_{3}\right)$ there exists $\lambda>0$, such that $G(x)+G(y) \geq \lambda G(x+y)$, for $x, y \in$ $\mathbb{R}$ and $x, y>0$,

$\left(A_{4}\right) \alpha(\beta(t))=\beta(\alpha(t))$ for every right - scattered point $t \in\left[t_{0}, \infty\right)_{\mathbb{T}}, t_{0} \geq$ 0 .

REMARK 1. The prototype of $G$ satisfying $\left(A_{1}\right),\left(A_{2}\right)$ and $\left(A_{3}\right)$ could be of the form

$$
\begin{gathered}
G(u)=\left(a^{*}+b^{*}|u|^{\gamma}\right)|u| \text { sgnu }, \\
G(u)=|u|^{\eta} \text { sgnu, }
\end{gathered}
$$

where $a^{*} \geq l, b^{*} \geq 1, \gamma \geq 0$ and $\eta>0$.

Indeed, the credit goes to S. Hilger [11], who has developed the concept of time scales in his Ph.D work. The purpose of the time scales was not only to unify the continuous and discrete aspects of mathematics, but also some cases in between. The study of dynamic equations on time scales allows us to avoid proving results twice, once for differential equations and once for difference equations. Our general idea is to prove a result for the dynamic equation (1.3), where the domain of the unknown function is a time scale $\mathbb{T}$ which is a non-empty closed subset of the real numbers $\mathbb{R}$. In this way, the results of this paper not only apply to $\mathbb{T}=\mathbb{R}$ and $\mathbb{T}=\mathbb{Z}$ for the subsequent equations 


$$
\begin{gathered}
\left(p(t)\left((u(t)+r(t) u(\alpha(t)))^{\prime \prime}\right)^{\prime \prime}+g(t) G(u(\beta(t)))-\right. \\
-h(t) H(u(\beta(t)))=0
\end{gathered}
$$

and

$$
\begin{aligned}
\Delta^{2}\left(p ( n ) \left(\Delta^{2}(u(n)\right.\right. & +r(n) u(\alpha(n))))+g(n) G(u(\beta(n)))- \\
& -h(n) H(u(\beta(n)))=0
\end{aligned}
$$

but also to more general time scales such as $\mathbb{T}=h \mathbb{N}, \mathbb{T}=q^{\mathbb{N}_{0}}=\{t: t=$ $\left.q^{k}, k \in \mathbb{N}_{0}\right\}$ with $q>1$ (which has important applications in quantum theory [12]), $\mathbb{T}=\mathbb{N}_{0}^{2}=\left\{t^{2}: t \in \mathbb{N}_{0}\right\}, \mathbb{T}=\left\{\sqrt{n}: n \in \mathbb{N}_{0}\right\}$ etc. For basic notations on time scales and time scale calculus, we refer the survey paper [1] by Agarwal and the monographs $[4,5]$ by Bohner et al.

In electric networks, we find the application of neutral delay differential equations due to high speed computers concerning the lossless transmission lines(see for e.g.[10]). So, we are interested in the problem to obtain sufficient conditions in order to ensure that the nonlinear neutral delay dynamic equation (1.3) is oscillatory. We may note that, (1.3) includes a class of differential and difference equations with delay argument of neutral/nonneutral type. In comparison with the present work, we refer the reader to some of the works $[7,13,17,18,19,20,21]$ on dynamic equations and the references cited there in. More on oscillation of unbounded solutions when $\mathbb{T}=\mathbb{R}$, we refer the monograph by Berezansky et al. [3] and some related works [6], [2]. Since we are interested in the oscillatory behaviour of solutions near infinity, we assume that $\sup \mathbb{T}=\infty$, and define the time scale interval $\left[t_{0}, \infty\right)_{\mathbb{T}}=\left[t_{0}, \infty\right) \cap \mathbb{T}$.

Let $t_{-1}=i n f_{t \in\left[t_{0}, \infty\right)_{\mathbb{T}}}\{\alpha(t), \beta(t)\}$. By a solution of (1.3) we mean a nontrivial real valued function $u$ on $\left[T_{u}, \infty\right)_{\mathbb{T}}$ such that $(u(t)+r(t) u(\alpha(t)) \in$ $C_{r d}^{2}(\mathbb{T}, \mathbb{R}),\left(p(t)(u(t)+r(t) u(\alpha(t)))^{\Delta^{2}}\right) \in C_{r d}^{2}(\mathbb{T}, \mathbb{R})$ and satisfies (1.3) for $T_{u} \geq t_{-1}>t_{0}>0$. In this paper, we do not consider the solutions that eventually vanish identically. A solution $u$ of (1.3) is said to be oscillatory, if it is neither eventually positive nor eventually negative and it is nonoscillatory otherwise.

2. Some Preparatory Results. In this section, we present some preparatory results which are useful for our discussion. Throughout our text, we use the quasi-derivative operators on $\mathbb{T}$ as follows:

$L_{0} u=u, L_{1} u=L_{0} u^{\Delta}, L_{2} u=p(t) L_{1} u^{\Delta}, L_{3} u=L_{2} u^{\Delta}, L_{4} u=L_{3} u^{\Delta}$.

LEMma 1. [9] Let $\left(A_{00}\right)$ hold. Let $u$ be a real valued function on $\left[t_{0}, \infty\right)_{\mathbb{T}}$ such that $L_{4} u(t) \leq 0$ for large $t$. If $u(t)>0$ ultimately, then one of cases (a) and (b) holds for large $t$, and if $u(t)<0$ ultimately, then one of cases (b), (c), (d) and (e) holds for large $t$, where

(a) $L_{1} u(t)>0, L_{2} u(t)>0$ and $L_{3} u(t)>0$, 
(b) $L_{1} u(t)>0, L_{2} u(t)<0$ and $L_{3} u(t)>0$,

(c) $L_{1} u(t)<0, L_{2} u(t)<0$ and $L_{3} u(t)>0$,

(d) $L_{1} u(t)<0, L_{2} u(t)<0$ and $L_{3} u(t)<0$,

(e) $L_{1} u(t)<0, L_{2} u(t)>0$ and $L_{3} u(t)>0$.

Lemma 2. [20] Let $\left(A_{0}\right)$ hold. Let $u$ be a real valued function on $\left[t_{0}, \infty\right)_{\mathbb{T}}$ such that $L_{4} u(t) \leq 0$ for large $t$. If $u(t)>0$ ultimately, then one of cases (a)-(d) holds for large $t$, and if $u(t)<0$ ultimately, then one of cases $(b)-(f)$ holds for large $t$, where

(a) $L_{1} u(t)>0, L_{2} u(t)>0$ and $L_{3} u(t)>0$,

(b) $L_{1} u(t)>0, L_{2} u(t)<0$ and $L_{3} u(t)>0$,

(c) $L_{1} u(t)>0, L_{2} u(t)<0$ and $L_{3} u(t)<0$,

(d) $L_{1} u(t)<0, L_{2} u(t)>0$ and $L_{3} u(t)>0$,

(e) $L_{1} u(t)<0, L_{2} u(t)<0$ and $L_{3} u(t)>0$,

(f) $L_{1} u(t)<0, L_{2} u(t)<0$ and $L_{3} u(t)<0$.

3. Unbounded Oscillation Results. In this section, unbounded oscillation criteria for (1.3) are established under the assumptions $\left(A_{0}\right)$ and $\left(A_{00}\right)$. Before stating our main results, we assume the following hypotheses for our use in the sequel:

$$
\begin{gathered}
B[s, v]=\int_{v}^{s} \frac{(s-\sigma(t))(t-v)}{p(t)} \Delta t, s>\sigma(t)>t>v, \\
C[v, u]=\int_{u}^{v} \frac{(\sigma(t)-u)(v-t)}{p(t)} \Delta t, v>\sigma(t)>t>u, \\
D[v, u]=\int_{u}^{v} \frac{(\sigma(t)-u)(t-u)}{p(t)} \Delta t, v>\sigma(t)>t>u .
\end{gathered}
$$

THEOREM 1. Let there exist $0<a<\infty$ such that $0 \leq r(t) \leq a<\infty$ for $t \in\left[t_{0}, \infty\right)_{\mathbb{T}}$. Assume that $\left(A_{0}\right)-\left(A_{4}\right)$ hold. Furthermore, assume that

$\left(A_{5}\right) \int_{T}^{\infty} \frac{\sigma(s)}{p(s)} \int_{s}^{\infty} \sigma(t) h(t) \Delta t \Delta s<\infty, T>0$

$\left(A_{6}\right) \frac{G(x)}{x} \geq M_{1}>0, x \neq 0$,

$\left(A_{7}\right) \lim \sup _{s \rightarrow \infty} \int_{\beta(s)}^{s} Q(\theta) G[B(\beta(\theta), \beta(s))] \Delta \theta>\frac{1+G(a)}{\lambda M_{1}}$,

$\left(A_{8}\right) \lim \sup _{t \rightarrow \infty} \int_{\beta(t)}^{t} Q(\theta) G[C(\beta(\theta), \beta(t))] \Delta \theta>\frac{1+G(a)}{\lambda M_{1}}$,

$\left(A_{9}\right) \lim \sup _{\theta \rightarrow \infty} \int_{\beta^{2}(\theta)}^{\beta(\theta)} Q(v) G[D(\beta(v), \beta(\theta))] \Delta v>\frac{(1+G(a))}{\lambda M_{1}}$

and

$\left(A_{10}\right) \lim \sup _{s \rightarrow \infty} \int_{\beta(s)}^{s} Q(\theta) G[D(\beta(s), \beta(\theta))] \Delta \theta>\frac{(1+G(a))}{\lambda M_{1}}$

hold, where $Q(t)=\min \{g(t), g(\alpha(t))\}$ for $t \geq t_{-1}$. Then every unbounded solution of (1.3) oscillates.

Proof. On the contrary, let $u(t)$ be an unbounded non-oscillatory solution of $(1.3)$ on $\left[t_{0}, \infty\right)_{\mathbb{T}}$. Without loss of generality and due to $\left(A_{2}\right)$, we can find a $t_{1} \in\left[t_{0}, \infty\right)_{\mathbb{T}}$ sufficiently large such that $u(t)>0, u(\alpha(t)), u(\beta(t)), u(\gamma(t))>0$ on $\left[t_{1}, \infty\right)_{\mathbb{T}}$. Setting

$$
z(t)=u(t)+r(t) u(\alpha(t))
$$




$$
\begin{gathered}
K(t)=\int_{t}^{\infty} \frac{(\sigma(s)-t)}{p(s)} \int_{s}^{\infty}(\sigma(\theta)-s) h(\theta) H(u(\gamma(\theta))) \Delta \theta \Delta s, \\
m(t)=z(t)-K(t)=u(t)+r(t) u(\alpha(t))-K(t)
\end{gathered}
$$

in (1.3), we get

$$
L_{4} m(t)=-g(t) G(u(\beta(t))) \leq 0 .
$$

Therefore, there exists a $t_{2} \in\left[t_{1}, \infty\right)_{\mathbb{T}}$ such that $L_{i} m(t), i=0,1,2,3$ are eventually of one sign on $\left[t_{2}, \infty\right)_{\mathbb{T}}$. Assume that $L_{0} m(t)>0$ and hence $m(t) \leq z(t)$. In what follows, we consider Cases $(a)-(d)$ of Lemma 2.

Case(a) For $x \geq y>t_{2}$,

$$
L_{2} m(x)-L_{2} m(y)=\int_{y}^{x} L_{3} m(s) \Delta s \geq(x-y) L_{3} m(x)
$$

implies that $L_{2} m(x) \geq(x-y) L_{3} m(x)$, that is,

$$
m^{\Delta^{2}}(x) \geq \frac{(x-y)}{p(x)} L_{3} m(x) .
$$

For $s>\sigma(t)>t>t_{2}$, we have the Taylor's formula

$$
m(s)-m\left(t_{2}\right)-\left(s-t_{2}\right) m^{\Delta}\left(t_{2}\right)=\int_{t_{2}}^{s}(s-\sigma(t)) m^{\Delta^{2}}(t) \Delta t .
$$

Therefore,

$$
\begin{gathered}
m(s)>\int_{t_{2}}^{s}(s-\sigma(t)) m^{\Delta^{2}}(t) \Delta t \geq \\
\geq \int_{t_{2}}^{s} \frac{(s-\sigma(t))(t-y)}{p(t)} L_{3} m(t) \Delta t \geq \\
\geq L_{3} m(s) \int_{y}^{s} \frac{(s-\sigma(t))(t-y)}{p(t)} \Delta t= \\
=L_{3} m(s) B[s, y], s>y \geq t_{2}
\end{gathered}
$$

due to (3.2). Letting $s=\beta(\theta)$ and $y=\beta(s)$ in the preceding relation, we obtain

$$
m(\beta(\theta)) \geq L_{3} m(\beta(\theta)) B[\beta(\theta), \beta(s)], \beta(\theta)>\beta(s) \geq t_{2}
$$

In addition to (1.3), it is easy to see that

$$
\begin{gathered}
0=L_{4} m(t)+g(t) G(u(\beta(t)))+G(a) L_{4}(\alpha(t))+G(a) g(\alpha(t)) G(u(\beta(\alpha(t)))) \geq \\
\geq L_{4} m(t)+G(a) L_{4} m(\alpha(t))+Q(t)[G(u(\beta(t)))+G(a) G(u(\alpha(\beta(t))))] \geq \\
\geq L_{4} m(t)+G(a) L_{4} m(\alpha(t))+\lambda Q(t) G(z(\beta(t))) \geq \\
\geq L_{4} m(t)+G(a) L_{4} m(\alpha(t))+\lambda Q(t) G(m(\beta(t)))
\end{gathered}
$$


due to $\left(A_{1}\right),\left(A_{3}\right),\left(A_{4}\right)$ and $z(t) \leq u(t)+a u(\alpha(t))$. Applying (3.3) in the last inequality, it follows that

$$
\begin{aligned}
& 0 \geq L_{4} m(\theta)+G(a) L_{4} m(\alpha(\theta))+\lambda Q(\theta) G\left(L_{3} m(\beta(\theta)) B[\beta(\theta), \beta(s)]\right) \geq \\
& \geq L_{4} m(\theta)+G(a) L_{4} m(\alpha(\theta))+\lambda Q(\theta) G\left(L_{3} m(\beta(\theta))\right) G(B[\beta(\theta), \beta(s)]) .
\end{aligned}
$$

Integrating the above inequality from $\beta(s)$ to $s$, we obtain

$$
\lambda \int_{\beta(s)}^{s} Q(\theta) G\left(L_{3} m(\beta(\theta))\right) G(B[\beta(\theta), \beta(s)]) \Delta \theta \leq L_{3} m(\beta(s))+G(a) L_{3} m(\alpha(\beta(s))),
$$

that is,

$$
\lambda G\left(L_{3} m(\beta(s))\right) \int_{\beta(s)}^{s} Q(\theta) G(B[\beta(\theta), \beta(s)]) \Delta \theta \leq(1+G(a)) L_{3} m(\alpha(\beta(s))),
$$

where $\alpha(\beta(s)) \leq \beta(s)$. As a result,

$$
\begin{gathered}
\int_{\beta(s)}^{s} Q(\theta) G(B[\beta(\theta), \beta(s)]) \Delta \theta \leq \\
\leq \frac{(1+G(a)) L_{3} m(\alpha(\beta(s)))}{\lambda G\left(L_{3} m(\beta(s))\right)} \leq \\
\leq \frac{(1+G(a))}{\lambda M_{1}}
\end{gathered}
$$

due to $\left(A_{6}\right)$, a contradiction to $\left(A_{7}\right)$.

Case(b) For $y>\sigma(t)>t>x \geq t_{2}$, we have the Taylor's formula

$$
\begin{gathered}
-m(y)=-m(x)-(y-x) m^{\Delta}(y)+\int_{x}^{y}(\sigma(t)-x) m^{\Delta^{2}}(t) \Delta t \leq \\
\leq \int_{x}^{y}(\sigma(t)-x) m^{\Delta^{2}}(t) \Delta t .
\end{gathered}
$$

Following to Case $(a)$, it is easy to see that $-L_{2} m(x) \geq(y-x) L_{3} m(y)$, that is, $-m^{\Delta^{2}}(x) \geq \frac{(y-x)}{p(x)} L_{3} m(y)$. Therefore,

$$
\begin{aligned}
& m(y) \geq \int_{x}^{y} \frac{(\sigma(t)-x)(y-t)}{p(t)} L_{3} m(y) \Delta t= \\
= & L_{3} m(y) C[y, x], y \geq s>\sigma(t)>t>x \geq t_{2} .
\end{aligned}
$$

Letting $y=\beta(\theta)$ and $x=\beta(t)$ in the preceding relation, we obtain

$$
m(\beta(\theta)) \geq L_{3} m(\beta(\theta)) C[\beta(\theta), \beta(t)], \beta(\theta)>\beta(t) \geq t_{2} .
$$

The rest of this case follows from Case $(a)$.

Case(c) From (3.4), we have

$$
-m(y) \leq \int_{x}^{y}(\sigma(t)-x) m^{\Delta^{2}}(t) \Delta t .
$$


Further,

$$
L_{2} m(y)-L_{2} m(x)=\int_{x}^{y} L_{3} m(s) \Delta s \leq(y-x) L_{3} m(x)
$$

implies that $L_{2} m(y) \leq(y-x) L_{3} m(x)$, that is, $m^{\Delta^{2}}(y) \leq \frac{(y-x)}{p(y)} L_{3} m(x)$. Consequently,

$$
\begin{gathered}
m(y) \geq \int_{x}^{y}(\sigma(t)-x) \frac{(t-x)}{p(t)}\left(-L_{3} m(x)\right) \Delta t= \\
=\left(-L_{3} m(x)\right) D[y, x]
\end{gathered}
$$

for $y \geq s>\sigma(t)>t>x \geq t_{2}$. Letting $y$ and $x$ by $\beta(y)$ and $\beta(\theta)$ respectively in the last inequality, we get

$$
m(\beta(y))>\left(-L_{3} m(\beta(\theta))\right) D[\beta(y), \beta(\theta)]
$$

for $\beta(y) \geq s>\sigma(t)>t>\beta(\theta) \geq t_{2}$. Proceeding as in Case $(a)$, we have

$$
0 \geq L_{4} m(t)+G(a) L_{4} m(\alpha(t))+\lambda Q(t) G(m(\beta(t)))
$$

which on applying (3.5), we find

$$
0 \geq L_{4} m(y)+G(a) L_{4} m(\alpha(y))+\lambda Q(y) G\left(-L_{3} m(\beta(\theta))\right) G(D[\beta(y), \beta(\theta)])
$$

Integrating the above inequality from $\beta^{2}(\theta)$ to $\beta(\theta)$, we obtain

$$
\begin{gathered}
\lambda \int_{\beta^{2}(\theta)}^{\beta(\theta)} Q(y) G\left(-L_{3} m(\beta(\theta))\right) G[D(\beta(y), \beta(\theta))] \Delta y \leq-L_{3} m(\beta(\theta))-G(a) L_{3} m(\alpha(\beta(\theta))) \leq \\
\leq-(1+G(a)) L_{3} m(\beta(\theta)) .
\end{gathered}
$$

As a result,

$$
\int_{\beta^{2}(\theta)}^{\beta(\theta)} Q(y) G[D(\beta(y), \beta(\theta))] \Delta y \leq \frac{(1+G(a))}{\lambda} \frac{\left(-L_{3} m(\beta(\theta))\right)}{G\left(-L_{3} m(\beta(\theta))\right)} \leq \frac{(1+G(a))}{\lambda M_{1}}
$$

a contradiction to $\left(A_{9}\right)$.

Case(d) From (3.4), we have

$$
z(x) \geq \int_{x}^{y}(\sigma(t)-x) m^{\Delta^{2}}(t) \Delta t
$$

Since

$$
L_{2} m(y)-L_{2} m(x)=\int_{x}^{y} L_{3} m(s) \Delta s \geq(y-x) L_{3} m(y)
$$


then $L_{2} m(y) \geq(y-x) L_{3} m(y)$, that is, $m^{\Delta^{2}}(y) \geq \frac{(y-x)}{p(y)} L_{3} m(y)$ and

$$
\begin{gathered}
m(x) \geq \int_{x}^{y}(\sigma(t)-x) \frac{(t-x)}{p(t)} L_{3} m(t) \Delta t \geq \\
\geq L_{3} m(y) \int_{x}^{y}(\sigma(t)-x) \frac{(t-x)}{p(t)} \Delta t= \\
=L_{3} m(y) D[y, x] .
\end{gathered}
$$

Letting $x=\beta(\theta)$ and $y=\beta(s)$, we get

$$
m(\beta(\theta)) \geq L_{3} m(\beta(s)) D[\beta(s), \beta(\theta)],
$$

for $\beta(s)>\beta(\theta) \geq t_{2}$. Using (3.7) in (3.6) it follows that

$$
0 \geq L_{4} m(\theta)+G(a) L_{4} m(\alpha(\theta))+\lambda Q(\theta) G\left(L_{3} m(\beta(s))\right) G(D[\beta(s), \beta(\theta)]) .
$$

Integrating the above inequality from $\beta(s)$ to $s$, we obtain

$$
\begin{gathered}
\lambda \int_{\beta(s)}^{s} Q(\theta) G\left(L_{3} m(\beta(s))\right) G[D(\beta(s), \beta(\theta))] \Delta \theta \leq L_{3} m(\beta(s))+G(a) L_{3} m(\alpha(\beta(s))) \leq \\
\leq(1+G(a)) L_{3} m(\alpha(\beta)(s)),
\end{gathered}
$$

Consequently,

$\int_{\beta(s)}^{s} Q(\theta) G[D(\beta(s), \beta(\theta))] \Delta \theta \leq \frac{(1+G(a)) L_{3} m(\alpha(\beta)(s))}{\lambda G\left(L_{3} m(\beta(s))\right)} \leq \frac{(1+G(a))}{\lambda M_{1}}$,

a contradiction to our hypothesis $\left(A_{10}\right)$.

Let $m(t)<0$ for $t \geq t_{2}$. Than $m(t)=z(t)-K(t)<0$ implies that

$$
u(t) \leq z(t)=u(t)+r(t) u\left(\alpha(t)<K(t) \text { for } t \geq t_{2} .\right.
$$

It is noticed that $K(t)>0$ and $K^{\Delta}(t)<0$, then $z(t)$ is bounded and so also $u(t)$ which is a contradiction. Therefore, this case doesn't arise when $u(t)$ is unbounded. This completes the proof of the theorem.

THEOREM 2. Let $-1<r(t) \leq 0$ for $t \in\left[t_{0}, \infty\right)_{\mathbb{T}}$. Assume that $\left(A_{0}\right)-$ $\left(A_{2}\right),\left(A_{5}\right)$ and $\left(A_{6}\right)$ hold. Furthermore, assume that

$\left(A_{11}\right) \limsup _{s \rightarrow \infty} \int_{\beta(s)}^{s} g(\theta) G[B(\beta(\theta), \beta(s))] \Delta \theta>\frac{1}{M_{1}}$,

$\left(A_{12}\right) \lim \sup _{t \rightarrow \infty} \int_{\beta(t)}^{t} g(\theta) G[C(\beta(\theta), \beta(t))] \Delta \theta>\frac{1}{M_{1}}$,

$\left(A_{13}\right) \lim \sup _{\theta \rightarrow \infty} \int_{\beta^{2}(\theta)}^{\beta(\theta)} g(v) G[D(\beta(v), \beta(\theta))] \Delta v>\frac{1}{M_{1}}$,

$\left(A_{14}\right) \lim \sup _{s \rightarrow \infty} \int_{\beta(s)}^{s} g(\theta) G[D(\beta(s), \beta(\theta))] \Delta \theta>\frac{1}{M_{1}}$

and

$\left(A_{15}\right) \quad \alpha^{n}(t)=\alpha\left(\alpha^{n-1}(t)\right), \lim _{n \rightarrow \infty} \alpha^{n}(t)<\infty$

hold. Then every unbounded solution of (1.3) oscillates. 
Proof. Suppose on the contrary that $u(t)$ is an unbounded nonoscillatory solution of (1.3) such that $u(t)>0, u(\alpha(t))>0, u(\beta(t))>0, u(\gamma(t))>0$ for $t \geq t_{0}>$. The case $u(t)<0$ can similarly be dealt with due to $\left(A_{2}\right)$. Proceeding as in Theorem 1, we get (3.1). So, there exists a $t_{1}>t_{0}$ such that $L_{i} m(t)$ for $i=0,1,2,3$ are eventually of one sign on $\left[t_{1}, \infty\right)$. Assume that $m(t)>0$ for $t \geq t_{2}$. Then, $m(t) \leq u(t)$ for $t \geq t_{2}$ and hence (1.3) can be written as

$$
L_{4} m(t)+g(t) G(m(\beta(t))) \leq 0, t \geq t_{2} .
$$

Ultimately, any one of four Cases $(a)-(d)$ of Lemma 2 holds for $t \geq t_{2}$. With (3.8), we can apply the arguments of the cases of Theorem 1 to get contradictions to $\left(A_{11}\right)-\left(A_{14}\right)$. Therefore, $m(t)<0$ for $t \geq t_{2}$, that is, $z(t)<K(t)$ for $t \geq t_{2}$ implies that $z(t)$ is bounded and hence $m(t)$ is bounded for $t \geq t_{2}$. Since $m(t)$ is monotonic, then $\lim _{t \rightarrow \infty} m(t)$ exists. On the other hand, $u(t)$ is unbounded and therefore it suggests that either $u(t)>u(\alpha(t))$ or $u(t)<u(\alpha(t))$ for $t \geq t_{2}$. If the former holds, then

$m(t)=u(t)+r(t) u(\alpha(t))-K(t)>(1+r(t)) u(\alpha(t))-K(t) \rightarrow \infty$ as $t \rightarrow \infty$

gives a contradiction. If the later holds, then

$$
u(t)<u(\alpha(t))<u\left(\alpha^{2}(t)\right)<\ldots .<u\left(\alpha^{n}(t)\right)<\ldots,
$$

leads to the fact that $u(t)$ is bounded due to $\left(A_{15}\right)$ which is absurd. Hence, the theorem is proved.

Theorem 3. Let $-\infty<r(t)<-1$ for $t \in\left[t_{0}, \infty\right)_{\mathbb{T}}$. Assume that all conditions of Theorem 2 hold. If

$\left(A_{16}\right) \quad \int_{0}^{\infty} g(t) d t=\infty$,

then every unbounded solution of (1.3) oscillates.

Proof. On the contrary, we proceed as in the proof of Theorem 2 and the case $m(t)>0$ is similar. When $m(t)<0$ for $t \geq t_{2}$, we have that $z(t)<K(t)$ for $t \geq t_{2}$, that is, $z(t)$ is bounded and thus $m(t)$ is bounded for $t \geq t_{2}$. Because $m(t)$ is monotonic, $\lim _{t \rightarrow \infty} m(t)$ exists. Here, we consider Cases $(b)-(f)$ of Lemma 2.

Cases (b), (d), (e) Since $u(t)$ is unbounded, then either $u(t)>u(\alpha(t))$ or $u(t)<u(\alpha(t))$ for $t \geq t_{2}$. Suppose that $u(t)<u(\alpha(t))$ for $t \geq t_{2}$. Then

$m(t)=u(t)+r(t) u(\alpha(t))-K(t)<(1+r(t)) u(\alpha(t))-K(t) \rightarrow-\infty$ as $t \rightarrow \infty$

which gives a contradiction to the existence of the limit. Hence, $u(t)>$ $u(\alpha(t))$ implies that

$$
u(t)>u(\alpha(t))>u\left(\alpha^{2}(t)\right)>\ldots .>u\left(\alpha^{n}(t)\right)>\ldots,
$$

that is, $\liminf _{t \rightarrow \infty} u(t)>0$. Let there exist a $t_{3}>t_{2}$ and $\eta>0$ such that $u(\beta(t))>\eta$ for $t \geq t_{3}$. Integrating (3.1) from $t_{3}$ to $\infty$, we get a 
contradiction to $\left(A_{16}\right)$. In Cases $(c)$ and $(f)$, it is immediate to see that $\lim _{t \rightarrow \infty} L_{1} m(t)=-\infty$ and $\lim _{t \rightarrow \infty} L_{0} m(t)=-\infty$, that is, these cases are not possible. Hence, the proof of the theorem is complete.

Theorem 4. Let there exist $0<a<\infty$ such that $0 \leq r(t) \leq a<\infty$ for $t \in\left[t_{0}, \infty\right)_{\mathbb{T}}$. Assume that $\left(A_{00}\right)$ and $\left(A_{1}\right)-\left(A_{8}\right)$ hold. Then every unbounded solution of (1.3) oscillates.

Proof. The proof of the theorem follows from Lemma 1 and Theorem 1. Hence, the details are omitted.

TheOREM 5. Let $-1<r(t) \leq 0$ for $t \in\left[t_{0}, \infty\right)_{\mathbb{T}}$. If $\left(A_{00}\right),\left(A_{1}\right),\left(A_{2}\right),\left(A_{5}\right),\left(A_{6}\right),\left(A_{11}\right)$ and $\left(A_{12}\right)$ hold, then every unbounded solution of (1.3) oscillates.

Proof. The proof of the theorem follows from Lemma 1 and Theorem 2 and hence the details are omitted.

Theorem 6. Let $-\infty<r(t)<-1$ for $t \in\left[t_{0}, \infty\right)_{\mathbb{T}}$. Assume that all conditions of Theorem 5 hold. If $\left(A_{16}\right)$ hold, then every unbounded solution of (1.3) oscillates.

Proof. The proof of the theorem follows from Theorem 5 and Theorem 3 and hence the details are omitted.

REMARK 2. In this work, we didn't discuss about the existence of nonoscillatory solutions of (1.3). However, the results of [9] and [16] are taken into account.

REMARK 3. It would be interesting to apply the results of this work to the following nonlinear dynamic equations:

$$
\left(p(t)\left((u(t)+r(t) u(\alpha(t)))^{\Delta^{2}}\right)\right)^{\Delta^{2}}+\sum_{i=1}^{n}(-1)^{i+1} g_{i}(t) G_{i}\left(u\left(\beta_{i}(t)\right)\right)=0 .
$$

Example. Let $\mathbb{T}=\mathbb{R}$ and consider Theorem 5. In this case,

$$
\begin{gathered}
\frac{(s-t)(t-v)}{p(t)} d t, s>t>v, \\
C[v, u]=\int_{u}^{v} \frac{(t-u)(v-t)}{p(t)} d t, v>t>u,
\end{gathered}
$$

that is, $B[s, v]=C[v, u]$, and $\left(A_{5}\right),\left(A_{11}\right),\left(A_{12}\right)$ reduce to

$$
\int_{T}^{\infty} \frac{s}{p(s)} \int_{s}^{\infty} t h(t) d t d s<\infty, T>0
$$

$\limsup _{s \rightarrow \infty} \int_{\beta(s)}^{s} g(\theta) G[B(\beta(\theta), \beta(s))] d \theta>\frac{1}{M_{1}}, \limsup _{t \rightarrow \infty} \int_{\beta(t)}^{t} g(\theta) G[C(\beta(\theta), \beta(t))] d \theta>\frac{1}{M_{1}}$, 
that is, $\left(A_{11}\right)=\left(A_{12}\right)$ respectively. Consider

$$
\left.\left(e^{-t}(k(s+5))+\left(-\frac{1}{25} e^{\pi}\right) u(t-\pi)\right)^{\prime \prime}\right)^{\prime \prime}+g(t) G\left(u\left(t-\frac{3 \pi}{2}\right)\right)-h(t) H\left(u\left(t-\frac{\pi}{2}\right)\right)=0
$$

for $t \in\left[\frac{\pi}{2}, \infty\right)$, where $\alpha(t)=t-\pi, \beta(t)=t-\frac{3 \pi}{2}, \gamma(t)=t-\frac{\pi}{2}, G(u)=u$, $H(u)=\frac{u}{1+u^{2}}, g(t)=e^{\frac{3 \pi}{2}}\left(\frac{52}{25} e^{-t}-e^{-3 t}\right)$ and $h(t)=e^{\frac{\pi}{2}}\left(e^{-2 t}+e^{-\pi} \cos ^{2} t\right) e^{-t}$. Indeed,

$$
\int_{0}^{\infty} \frac{s}{p(s)} \int_{s}^{\infty} t h(t) d t d s=\int_{0}^{\infty} \frac{s}{e^{-s}} \int_{s}^{\infty} t\left[e^{\frac{\pi}{2}}\left(e^{-2 t}+e^{-\pi} \cos ^{2} t\right) e^{-t}\right] d t d s<\infty
$$

and

$$
\limsup _{s \longrightarrow \infty} \int_{s-\beta}^{s} B[\theta-\beta, s-\beta] g(\theta) d \theta>1
$$

in which

$$
B[s, v]=\int_{v}^{s} \frac{(s-t)(t-v)}{e^{-t}} d t .
$$

By the generalized Leibnitz formula, we notice that

$$
\frac{d B}{d s}=s e^{s}\left[1-\frac{1}{s}-\frac{v}{s}\right]+e^{v} \rightarrow \infty \text { as } s \rightarrow \infty .
$$

Hence, we can find a constant $C>0$ such that $B[s, v] \geq C e^{s}$. Therefore,

$$
\begin{gathered}
\int_{s-\beta}^{s} B[\theta-\beta, s-\beta] g(\theta) d \theta \\
\geq \geq C \int_{s-\beta}^{s} e^{\theta} e^{\frac{3 \pi}{2}}\left(\frac{52}{25} e^{-\theta}-e^{-3 \theta}\right) d \theta= \\
=C e^{\frac{3 \pi}{2}}\left[\frac{52 \beta}{25}+\frac{e^{-2 s}}{2}\left(1-e^{2 \beta}\right)\right]>1
\end{gathered}
$$

as $s \rightarrow \infty$. Clearly, all conditions of Theorem 5 are satisfied. Hence (3.9) is oscillatory. In particular, $u(t)=e^{t} \sin t$ is such an unbounded oscillatory solution of (3.9). Example. On $\mathbb{T}=\mathbb{Z}$, consider Theorem 2. In this case

$$
\begin{gathered}
B[s, v]=\sum_{t=v}^{s} \frac{(s-t-1)(t-v)}{p(t)}, s>t+1>v, \\
C[v, u]=\sum_{t=u}^{v} \frac{(t+1-u)(v-t)}{p(t)}, v>t>u \\
D[v, u]=\sum_{t=u}^{v} \frac{(t+1-u)(t-u)}{p(t)}, v>t>u .
\end{gathered}
$$


Let's consider a nonlinear neutral difference equation

$\left.\triangle^{2}\left(\left(\cos ^{\mathrm{d}} \Delta \mathrm{\partial}\right) u(t)+r(t) u(t-2)\right)\right)+g(t) u(t-1)-h(t) \frac{u(t-2)}{1+u^{2}(t-2)}=0, t \geq 3$,

where $M_{1}=1, r(t)=-1<-\frac{e}{e^{t}} \leq 0, p(t)=e^{t}, h(t)=e^{2} \frac{\left(e^{4}+e^{2 t}\right)}{(t+1) e^{4 t}}$ and $g(t)=e(e+1)^{2}\left(e^{2}+1\right)^{2} e^{t}+4 e\left(e^{-1}+1\right)^{2} e^{-t}-\frac{e}{(t+1) e^{4 t}}$. For (3.10), we have

$\left(A_{5}\right) \sum_{s=T}^{\infty} \frac{s+1}{p(s)} \sum_{t=s}^{\infty}(t+1) h(t)<\infty, T>0$,

$\left(A_{11}\right) \lim \sup _{s \rightarrow \infty} \sum_{\theta=s-1}^{s} g(\theta)[B(\theta-1, s-1)]>1$,

$\left(A_{12}\right) \lim \sup _{t \rightarrow \infty} \sum_{\theta=t-1}^{t} g(\theta)[C(\theta-1, t-1)]>1$,

$\left(A_{13}\right) \lim \sup _{\theta \rightarrow \infty} \sum_{v=\theta-2}^{\theta-1} g(v)[D(v-1, \theta-1)]>1$,

$\left(A_{14}\right) \lim \sup _{s \rightarrow \infty} \sum_{\theta=s-1}^{s} g(\theta)[D(s-1, \theta-1)]>1$.

Clearly,

$$
\sum_{s=T}^{\infty} \frac{s+1}{p(s)} \sum_{t=s}^{\infty}(t+1) h(t)=\sum_{s=1}^{\infty} \frac{s+1}{e^{s}} \sum_{t=s}^{\infty}(t+1) e^{2} \frac{\left(e^{4}+e^{2 t}\right)}{(t+1) e^{4 t}}<\infty .
$$

If we denote

$[B(\theta-1, s-1)]=\sum_{t=s-1}^{\theta-1} \frac{(\theta-t-2)(t-s+1)}{e^{t}}=\sum_{t=s}^{\theta-1} \frac{(\theta-t-2)(t-s+1)}{e^{t}}=B(\theta)($ say $)$

for $\theta-1>t+1$, then

$$
\begin{gathered}
\Delta B(\theta)=\sum_{t=s}^{\theta} \frac{(\theta-t-1)(t-s+1)}{e^{t}-\sum_{t=s}^{\theta-1} \frac{(\theta-t-2)(t-s+1)}{e^{t}} \geq} \\
\geq \sum_{t=s}^{\theta-1} \frac{(t-s+1)}{e^{t}}>0 .
\end{gathered}
$$

Therefore, we can find a constant $C_{1}>0$ such that $[B(\theta-1, s-1)] \geq C_{1}$. Similarly, we can find constants $C_{2}>0$ and $C_{3}>0$ such that $[C(\theta-1, t-$ $1)] \geq C_{2}$ and $[D(v-1, \theta-1)] \geq C_{3}$. Consequently,

$$
\begin{gathered}
\limsup _{s \rightarrow \infty} \sum_{\theta=s-1}^{s} g(\theta)[B(\theta-1, s-1)] \geq C_{1} \limsup _{s \rightarrow \infty} \sum_{\theta=s-1}^{s} g(\theta)= \\
=C_{1} \limsup _{s \rightarrow \infty} \sum_{\theta=s-1}^{s}\left[e(e+1)^{2}\left(e^{2}+1\right)^{2} e^{\theta}+4 e\left(e^{-1}+1\right)^{2} e^{-\theta}-\frac{e}{(\theta+1) e^{4 \theta}}\right]>1
\end{gathered}
$$

for every $C_{1}>0$. Also, it is easy to see that $\left(A_{12}\right),\left(A_{13}\right)$ and $\left(A_{14}\right)$ hold true for every $C_{2}, C_{3}>0$. As a result, (3.10) satisfies all conditions of Theorem 2. In particular, $u(t)=(-e)^{t}$ is such an unbounded oscillatory solution of (3.10). Acknowledgement The author is thankful to the three referees for their careful reading and valuable suggestions about the completion of this work in this format. 


\section{REFERENCES}

[1] R. P. Agarwal, M. Bohner, D. O'Regan,A. Peterson, Dynamicequations on time scales: A survey, J. Compu. Appl. Math., 141,(2002), 1-26.

[2] L. Berezansky, A. Domoshnitsky, R. Koplatadze, Oscillation, Stability and Asymptotic Properties for Second and Higher Order Functional Differential Equations, CRC Press, 2020.

[3] L. Berezansky, A. Domoshnitsky, Instability for a class of second order delay differential equations, Elect. J. Quali. Theory Diff. Equa., 2017 (2017), 1-14.

[4] M. Bohner, A. Peterson, Dynamic equations on Time scales : An Introduction with Applications, Birkhäuser, Boston, 2001.

[5] M. Bohner, A. Peterson, Advances in Dynamic Equations on Time scales : Birkhäuser, Boston, 2003.

[6] A. Domoshnitsky, Unboundedness of solutions and instability of second order equations with delayed argument, Differential and Integral Equations, 14 (2001), 559576.

[7] S. R. Grace, M. Bohner, S. Sun, Oscillation of fourth orderdynamic equations, Hacet.J. Math. Stat., 39 (2010), 454-453.

[8] S. R. Grace, A. Zafer, Oscillation of fourth-order nonlinear neutral delay dynamic equations, Hacet. J. Math. Stat., 44 (2015), 331-339.

[9] G. R. Graef, S. Panigrahi, P. R. Reddy, On oscillatory and asymptotic behaviour of fourth order nonlinear neutral delay dynamic equations with positive and negative coefficients, Math. Slovaca, 64 (2014), 347-366.

[10] J. K. Hale, Theory of Functional Differential Equations, Springer-Verlag, New York, 1977.

[11] S. Hilger, Analysis on measure chains-a unified approach to continuous and discrete calculus, Results in Mathematics, 2 (2006), 123-141.

[12] V. Kac, P. Cheung, Quantum calculus, Universitent, Springer - Verlag, New York, 2002.

[13] T. Li, E. Thandapani, S. Tang, Oscillation theorems for fourth order delay dynamic equations on time scales, Bull. Math. Anal. Appl., 3 (2011), 190-199.

[14] S. Panigrahi, P. Reddy, On oscillatory fourth order nonlinear neutral delay dynamic equations, Comp. Math. Appl., 62 (2011), 4258-4271.

[15] S. Panigrahi, P. R. Reddy, Oscillatory and asymptotic behaviour of fourth order nonlinear neutral delay dynamic equations, Dyn. Cont. Disc. Impl. Syst., Ser-A, 20 (2013), 143-163.

[16] S. Panigrahi, G. Graef, P. Reddy, Oscillation results for fourth order nonlinear neutral dynamic equations, Commu. Math. Anal., 15 (2013), 11-28.

[17] A. Tripathy, S. Panigrahi, P. Reddy, Oscillation of a class of fourth order functional dynamic equations, Functional Diff. Equs., 21 (2014), 91-108.

[18] A. Tripathy, S. Panigrahi, P. R. Reddy, Oscillation of nonlinear fourth order mixed neutral dynamic equations, Functional Diff. Equs., 21 (2015), 69-91.

[19] A.Tripathy, New oscillation criteria for fourth order neutral dynamic equations, Commu. Appl. Anal., 20 (2016), 13-24.

[20] A. K. Tripathy,vOscillation of a class neutral delay dynamic equations of fourth order, Glo. Stoch. Anal., 5 (2018), 15-29.

[21] C. Zhang,Oscillation results for fourth order nonlinear dynamic equations, Appl. Math. Lett., 25 (2012), 2058-2065. 